\title{
ON A THEOREM OF GLEASON
}

\section{CHEVAlLEy}

In a recent paper (Square roots in locally euclidean groups, Bull. Amer. Math. Soc. vol. 55 (1949) pp. 446-449), A. M. Gleason proved that, in a locally euclidean group $G$ which has no small subgroups, there exist neighborhoods $M$ and $N$ of the neutral element $e$ such that every element in $M$ has a unique square root in $N$. The author clearly considered this result to be a step towards proving that, in such a group, some neighborhood of $e$ is entirely filled by oneparameter subgroups of $G$. We shall establish here that he was justified in his expectation. As a matter of fact, our present proof requires only the local uniqueness of the square root, not its existence. This local uniqueness is established in the second part of the proof of Theorem 4 in Gleason's paper; and it may be observed that this part of the argument is independent of the assumption that the group be locally euclidean: it works equally well under the weaker assumption that the group be locally compact. We therefore have the following result:

LEMMA 1 (GLEASON). Let $G$ be a locally compact group in which there exists a neighborhood of the neutral element $e$ which does not contain any subgroup $\neq\{e\}$ of $G$; then there is a neighbourhood $V$ of $e$ in $G$ such that distinct elements of $V$ have distinct squares.

If we take $V$ to be compact, then $V$ is mapped topologically under the mapping $s \rightarrow s^{2}$.

Now we prove

Theorem 1. Let $G$ be a locally euclidean group. Assume that there exists a neighborhood of the neutral element $e$ of $G$ which does not contain any subgroup $\neq\{e\}$ of $G$. Then there exists a neighborhood $P$ of $e$ which has the following property: if $t \in P$, there exists a uniquely determined continuous homomorphism $x \rightarrow s_{t}(x)$ of the additive group of real numbers into $G$ such that $s_{t}(x) \in P$ for $-1 \leqq x \leqq 1$ and $s_{t}(1)=t ; s_{t}(x)$ is a continuous function of the pair $(t, x)$.

It follows from Lemma 1 that there exists a neighborhood $V$ of $e$ which has the following properties: $V$ is compact; $V=V^{-1} ; V$ is mapped topologically under the mapping $s \rightarrow s^{2} ; V$ does not contain any subgroup $\neq\{e\}$ of $G$; some open set containing $V$ is homeo-

Received by the editors October 21, 1949. 
morphic to an open solid sphere in a euclidean space. If $n$ is any integer $>0$, we denote by $Q_{n}$ the set of elements $u \in V$ such that $u^{m} \in V$ for $1 \leqq m \leqq 2^{n}$. If $u \in Q_{n}$, then we have also $u^{m} \in V$ for $-2^{n} \leqq m \leqq 2^{n}$. The set $Q_{n}$ is obviously closed, and therefore compact. The mapping $s \rightarrow s^{2}$ being topological on $V$, the mapping $u \rightarrow u^{2^{n}}$ is a homeomorphism of $Q_{n}$ with some compact subset $P_{n}$ of $V$. If $t \in P_{n}$, we denote by $f_{n}(t)$ the element $u$ of $Q_{n}$ such that $u^{2^{n}}=t ; f_{n}$ is a homeomorphism of $P_{n}$ with $Q_{n}$.

Let $\left(t_{n}\right)$ be a sequence of elements of $V$ which converges to an element $t$ of $V$. Assume that $t_{n} \in P_{n}$ for every $n$. Then we shall prove that there exists a homomorphism $x \rightarrow s(x)$ of the group of real numbers into $G$ such that $s(1)=t$ and $s(x) \in V$ for all $x \in[-1,+1]$. Let us select once and for all a nontrivial ultrafilter $\mathfrak{F}$ on the set of positive integers (that is, an ultrafilter which does not consist of all sets containing a certain integer). Let $x$ be any real number in $[-1,+1]$, and let $\left(m_{n}\right)$ be a sequence of integers such that $\left|m_{n}\right| \leqq 2^{n}$ for every $n$ and $\lim _{\mathfrak{F}} 2^{-n} m_{n}=x$. Since $V$ is compact, the limit $\lim _{\mathfrak{F}}\left(f_{n}\left(t_{n}\right)\right)^{m_{n}}$ exists. We shall see that this limit depends only on $x$, not on the choice of the sequence $\left(m_{n}\right)$. Let $\left(m_{n}^{\prime}\right)$ be any sequence which satisfies the same conditions as $\left(m_{n}\right)$; set $s=\lim _{\mathfrak{F}}\left(f_{n}\left(t_{n}\right)\right)^{m_{n}}$ and $s^{\prime}$ $=\lim _{\mathfrak{F}}\left(f_{n}\left(t_{n}\right)\right)^{m_{n}^{\prime}}$. Denote by $u$ the element $s^{-1} s^{\prime}$, and by $k$ an arbitrary integer. Then, clearly, $u^{k}=\lim _{\mathfrak{F}}\left(f_{n}\left(t_{n}\right)\right)^{k\left(m_{n}^{\prime}-m_{n}\right)}$; on the other hand, we have $\lim _{\mathfrak{F}} 2^{-n} k\left(m_{n}^{\prime}-m_{n}\right)=0$. It follows that there exists a set $F$ in $\mathfrak{F}$ such that $\left|k\left(m_{n}^{\prime}-m_{n}\right)\right| \leqq 2^{n}$ for all $n \in F$; this implies that $\left(f_{n}(t)\right)^{k\left(m_{n}^{\prime}-m_{n}\right)}$ is in $V$ when $n \in F$, whence $u^{k} \in V$. Since $V$ does not contain any subgroup $\neq\{e\}$ of $G$, we have $u=e$, whence $s^{\prime}=s$. We denote by $s_{t}(x)$ the limit $\lim _{\mathfrak{F}}\left(f_{n}\left(t_{n}\right)\right)^{m_{n}}$. If $x, x^{\prime}$, and $x+x^{\prime}$ are in $[-1,+1]$, then it is easily seen that we can find sequences $\left(m_{n}\right)$ and $\left(m_{n}^{\prime}\right)$ such that $\left|m_{n}\right| \leqq 2^{n},\left|m_{n}^{\prime}\right| \leqq 2^{n},\left|m_{n}+m_{n}^{\prime}\right| \leqq 2^{n}$ and such that the sequences $\left(2^{-n} m_{n}\right)$ and $\left(2^{-n} m_{n}^{\prime}\right)$ converge (for instance in the ordinary sense) to $x$ and $x^{\prime}$; then the sequence $\left(2^{-n}\left(m_{n}+m_{n}^{\prime}\right)\right)$ converges to $x+x^{\prime}$, and it follows immediately that $s_{t}\left(x+x^{\prime}\right)=s_{t}(x) s_{t}\left(x^{\prime}\right)$. We conclude from this that the mapping $x \rightarrow s_{t}(x)$ may be extended in one and only one way to a homomorphism of the additive group of real numbers (cf., for instance, C. Chevalley, Theory of Lie groups, Theorem 3, chap. 2, §8, p. 49). It is clear that $s_{t}(1)=t$.

It is obvious that $Q_{p+1} \subset Q_{p}$ for every $p>0$. We shall prove that, given any neighborhood $N$ of $e$, we have $Q_{p} \subset N$ as soon as $p$ is large enough. We may assume $N$ to be open; the sets $Q_{p}-\left(Q_{p} \cap N\right)$ are then compact and they form a monotone decreasing sequence. Were not $Q_{p}$ contained in $N$ from a certain $p$ on, then there would exist a point $s$ in the intersection of all sets $Q_{p}-\left(Q_{p} \cap N\right)$; we would have $s \neq e$, 
but $s^{m} \in V$ for every integer $m$, which is impossible. Using the same notation as above, let us prove that $s_{t}(x) \in Q_{p}$ whenever $|x| \leqq 2^{-p}$. If $|x| \leqq 2^{-p}$, we can find a sequence $\left(m_{n}\right)$ such that $\lim \left(2^{-n} m_{n}\right)=x$ and $2^{-n}\left|m_{n}\right| \leqq 2^{-p}$ for every $n$. Since $\left|m_{n}\right| \leqq 2^{n-p}$, we have $\left(\left(\left(f_{n}\left(t_{n}\right)\right)^{\left.m_{n}\right)^{m}}\right.\right.$ $\in V$ for all integers $m$ such that $|m| \leqq 2^{p}$, and therefore $\left(f_{n}\left(t_{n}\right)\right)^{m_{n}} \in Q_{p}$, whence, since $Q_{p}$ is closed, $s_{t}(x) \in Q_{p}$.

Let $P$ be the intersection of the sets $P_{n}(1 \leqq n<\infty)$. Then $s_{t}(x)$ is in particular defined whenever $t \in P$ (we take $t_{n}=t$ for every $n$ ). Since $s_{t}\left(x+x^{\prime}\right)=s_{t}(x) s_{t}\left(x^{\prime}\right)$ and $s_{t}(x) \in Q_{p}$ when $|x| \leqq 2^{-p}$, the mappings $x \rightarrow s_{t}(x)$ form a family of equicontinuous mappings of the additive group of real numbers into $G$. We have $s_{t}(1)=t$ and $s_{t}(x) \in V$ for $x \in[-1,+1]$. Now, let $t$ be any point of $P$ and $\sigma$ a continuous homomorphism of the additive group of real numbers into $G$ such that $\sigma(1)=t$ and $\sigma(x) \in V$ for $x \in[-1,+1]$. Then it is clear that $\sigma\left(2^{-n}\right) \in Q_{n}$ for every $n>0$, whence $\sigma\left(2^{-n}\right)=f_{n}(t)$ and therefore $\sigma\left(2^{-n} m_{n}\right)$ $=\left(f_{n}(t)\right)^{m_{n}}$ for every integer $m_{n}$. Let $x$ be in $[-1,+1]$, and let $\left(m_{n}\right)$ be a sequence such that $\lim 2^{-n} m_{n}=x,\left|m_{n}\right| \leqq 2^{n}$; then, we have $\sigma(x)$ $=\lim \sigma\left(2^{-n} m_{n}\right)=\lim \left(f_{n}\left(t_{n}\right)\right)^{m_{n}}=\lim _{\mathfrak{F}}\left(f_{n}\left(t_{n}\right)\right)^{m_{n}}=s_{t}(x)$. It follows immediately that $\sigma(x)=s_{t}(x)$ for every real number $x$. Now, let \& be any filter on $P$ which converges to $t$. Then, for any fixed integer $n$, we have $\lim _{\Theta} f_{n}\left(t^{\prime}\right)=f_{n}(t)$, whence $\lim \Leftrightarrow s_{t^{\prime}}\left(2^{-n} m\right)=s_{t}\left(2^{-n} m\right)$ for every integer $m$. This fact, together with the equicontinuity of the mappings $s_{t}$, shows that, when $t^{\prime}$ converges to $t$, the mapping $s_{t^{\prime}}$ converges to $s_{t}$, uniformly on every compact subset of the additive group $R$ of real numbers; this means that the mapping $(x, t) \rightarrow s_{t}(x)$ of $R \times P$ into $G$ is continuous. Moreover, it is clear that, if $t \in P$, then $s_{t}(x) \in P$ for all $x$ in $[-1,+1]$.

The assumption that $G$ is locally euclidean has not been used as yet (the argument would go through in any locally compact group which has no small subgroups). We shall use this assumption to prove that $P$ is a neighborhood of $e$. If $u$ is an interior point of $Q_{n}$, then $u^{2^{n}}$ is an interior point of $P_{n}$. For, let $W$ be any neighborhood of $u$ in $Q_{n}$; the mapping $u \rightarrow u^{2^{n}}$ induces a homeomorphism of $W$ with a subset of $V$, and this subset is open in virtue of the theorem of invariance of the domain, which shows that $u^{2^{n}}$ is interior to $P_{n}$. Now, it is clear that, for every $n, Q_{n}$ is a neighborhood of $e$; it follows that $P_{n}$ is a neighborhood of $e$. Since $G$ is locally connected, in order to prove that $P$ is a neighborhood of $e$, it will be sufficient to prove that it is impossible to have a sequence $\left(t_{n}\right)$ of elements converging to $e$ such that each $t_{n}$ belongs to the boundary $B_{n}$ of $P_{n}$. Now, if $t_{n} \in B_{n}$, one at least of the points $\left(f_{n}\left(t_{n}\right)\right)^{m_{n}}\left(1 \leqq m_{n} \leqq 2^{n}\right)$ is on the boundary of $V$. For, were these points all interior to $V$, then, ob- 
viously, some neighborhood of $f_{n}\left(t_{n}\right)$ would be in $Q_{n}$, and $t_{n}$ would therefore be interior to $P_{n}$, as we have seen above. Let us then select a sequence $\left(m_{n}\right)$ such that $1 \leqq m_{n} \leqq 2^{n}$ and that $\left(f_{n}\left(t_{n}\right)\right)^{m_{n}}$ is on the boundary of $V$. The limit $x_{0}=\lim _{\mathfrak{F}} 2^{-n} m_{n}$ exists because the interval $[-1,+1]$ is compact. We may now apply the result proved above: there exists a homomorphism $x \rightarrow s(x)$ of the group of real numbers into $G$ such that $s\left(x_{0}\right)=\lim _{\mathfrak{F}}\left(f_{n}\left(t_{n}\right)\right)^{m_{n}}$ is on the boundary of $V, s(1)=\lim t_{n}=e$ and $s(x) \in V$ for $x \in[-1,+1]$. But then the set of elements $s(x)$, for all real $x$, is a group $\neq\{e\}$ contained in $V$, and we arrive at a contradiction. Theorem 1 is thereby proved.

Columbia University 